ISSN 1870-4069

\title{
System Identification of a Quad-rotor in X Configuration from Experimental Data
}

\author{
Victor Manuel Aboytes Reséndiz, Edgar Rivas Araiza \\ Universidad Autónoma de Querétaro, Querétaro, Mexico \\ victoraboytes@outlook.com
}

\begin{abstract}
Quad-rotors have been a very popular topic for research in the current literature because of its capability to perform different tasks autonomously. This paper focuses on the modeling of a quad-rotor aircraft dynamics based on experimental data. It will be demonstrated that a linear model could represent its dynamics despite of quadrotor's strong nonlinearities. Also, it will be shown how a quad-rotor aircraft system works, its typical mathematical model according with literature and a brief history of Unmanned Aerial Vehicles.
\end{abstract}

Keywords: Quadrotor, system identification, modeling, linear model.

\section{Introduction}

Technology has been evolving through the years and has given us the possibility to build and create complex machines. We are witnessing the new era of robots, where the use of quad-rotor machines, known as drones, in our daily lives has been on the increase. Thanks to technology, these aircrafts can handle many tasks autonomously in natural and man-made environments. Therefore, strong knowledge in the areas of electronics, control, and mechanics is important to understand the behavior of these aerial robotic systems.

\subsection{A Brief History of UAVs}

Contrary to many people's belief, drones or Unmanned Aerial Vehicles [1] (UAVs) are not a recent invention, going back a few decades. The UAVs have a rich history that goes back many years back and as a result of this, the human kind can use and explore the many possibilities that these rotorcrafts offer us.

The idea of building flying machines was first conceived around 2,500 years ago in ancient Greece and China. According to [2], the first known autonomous flying machine has been credited to Archytas from the city of Tarantas in southern Italy, also known as Archytas the Tarantine. In the same era, in China, in about $400 \mathrm{BC}$, the first document that showed the idea of a vertical flight aircraft was found.

Many centuries later, an aircraft, called aerial screw or air gyroscope [3], capable of hovering was designed by Leonardo Da Vinci in 1483. The first manned 
flight took place in 1783 using a hot air balloon designed by the Montgolfier brothers. As a result of these events, several flying machines were designed until the first helicopter was created in 1860s and later the fixed wing aircraft was introduced.

Today UAVs are more complex and reliable than before, thanks to the advancement of technology. Nowadays, aircrafts can manage different tasks [4], such as: inspection of structures, transportation of medical supplies, communication through network for rescue missions, and more.

\subsection{Related Work}

There are several kinds of methods to identify the unknown UAV's parameters considering their dynamics. Measurement of vehicle parameters, such as inertias, lift, and drag coefficients are commonly used to obtain nonlinear models. However, such values are difficult to obtain with a certain level of accuracy. Therefore, scientists have applied different methodologies to acquire UAV's parameters.

Genetic Algorithm (GA) is a globally optimal method for solving optimization problems such as quad-rotors system. Real-coded GA and Distributed Genetic Algorithm (DGA) [5-7] are used to determine the unknown parameters.

Least square method, maximum likelihood estimation, neural network identification, the fuzzy system identification, and wavelet network identification methods are used in identification of model [8,9]. Some examples of these identification methods are: ARX Model is an identification method to determine linear systems [10-12], RBF-ARX Model combine the linear ARX model structure with Radial Basis Function Neural Network (RBF) [13,14] and Fuzzy Modeling which describes the dynamic characteristics of complex and nonlinear dynamics.

\subsection{Motivation for System Identification}

The model of a quad-rotor commonly involves dynamic forces, momentums, and torques produced by the physical aircrafts elements such as propellers, sensing units, motors, airframes, and so on. Here lies the main problem when the rotorcraft's mathematical model is required. Different quad-rotor's elements, as mentioned before, change the dynamics and the parameters of a model and many of those parameters are difficult to obtain in an experiment.

Having an alternative method to obtain the quad-rotors model would improve the process of the design of the control algorithm without affecting the type of propellers, BLDC motors or airframe the aircraft has. This identification method has several advantages:

1. It reduces the amount of time wasted on obtaining the quad-rotor's parameters of the model in an experiment. This costs a lot of work and does not guarantee accuracy and can not detect important dynamics.

2. The algorithm can be used for modeling similar quad-rotor systems with larger or shorter propellers, bigger or smaller motors, different weight of the structure, and so on. 


\section{Quad-rotor}

There are different configurations for an aircraft depending on the number of motors. These configurations are: Tricopter, quadcopter, hexacopter, and octocopter. Another difference, beside the number of motors, is the shape of the frame. Therefore, there exists different variations of aircrafts even though the number of motors remains the same. The type of rotorcraft used in this paper is a quad-copter in $\mathrm{X}$ configuration.

\subsection{Quad-rotor Characteristics}

The quad-rotor rotorcraft is an aircraft with four rotors in a square. Due to this configuration, it is possible to hover, mover forward, sideward, up, down, and turn about the roll, pitch, and yaw axes separately. The four rotors spin clockwise and counter-clockwise like it is shown in Fig. 1. Clockwise (CW) motors, shown in blue, use normal propellers and counter-clockwise (CCW) motors, shown in red, use pusher propellers.

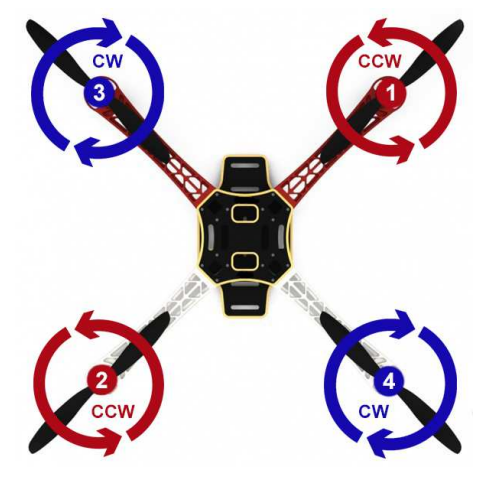

Fig. 1. Quad-rotor in X configuration.

This four-rotor aircraft has some advantages over typical helicopter. Due to rotation of the motors (clock and counter-clockwise), gyroscopic effects, and aerodynamic torques tend to cancel in flight.

Throttle input is the sum of the thrust of each motor (motors 1,2,3, and 4). This force makes the quad-copter fly. Roll movement is obtained by increasing or reducing the speed of rear motors (motors 2 and 4) and reducing or increasing front motors (motors 3 and 1) respectively. Pitch movement is obtained similarly using lateral motors. Lastly, yaw movement is obtained by increasing or decreasing the speed of the counter-clockwise motors while decreasing or increasing the speed of the clockwise motors. Figure 2 shows the UAV's angles of rotation. 


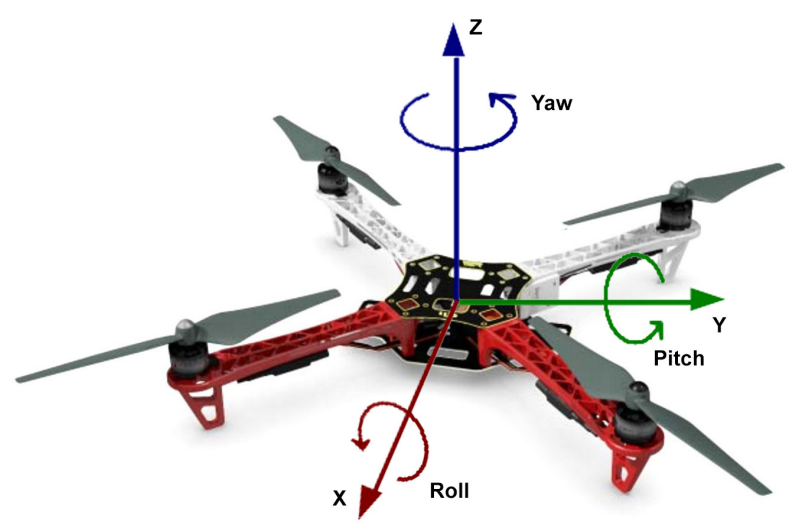

Fig. 2. Quad-rotor angles of rotation.

\subsection{Quad-rotor System}

A description of the quad-rotor used for the experiments:

1. Quad-rotor frame: The quad-rotor consist of a rigid body with 4 arms mounted in X configuration. A motor is mounted at the end of each arm.

2. Motors: Each rotor consists of a brushless motor (BLDC) and a propeller. Each of the motors are controlled by Electronic Speed Controllers (ESC). The input of an ESC is a Pulse Width Modulated signal. This signal is measured for the purpose of system identification on later stages.

3. Microcontroller Unit (MCU): A MCU is mounted on the central body of the quad-rotor. The MCU used is the Pixhawk which basically a $32 \mathrm{bit}$ STM32F427 Cortex M4 core with FPU. The Pixhawk hardware is integrated with different sensors like: 3-axis accelerometer/gyroscope, magnetometer, and barometer. It has different communication protocols such as: UART, CAN, $\mathrm{I}^{2} \mathrm{C}$, SPI. This MCU was selected because it is an open source project and we can design and program our own code using the Pixhawk.

\section{Quad-rotor Dynamic Model}

The dynamic model of the quad-rotor is firstly formulated in this section. Then a model based on experimental data will be obtained.

\subsection{Rigid Body Dynamics}

Quad-rotor's dynamics are represented by two reference frames: earth fixed $O_{E}$ and body fixed $O_{B}$ frame. See Fig. 3. $O_{E}$ is the fixed frame to the earth where $X$ points to the south, $Y$ points to the west, and $Z$ point up. On the other 
hand, $O_{B}$ is the fixed frame of the quad-rotor's body whose $x, y$, and $z$ are defined along the axes of the quadcopter. As a consequence 12 variables define the dynamical system of the quad-rotor [15].

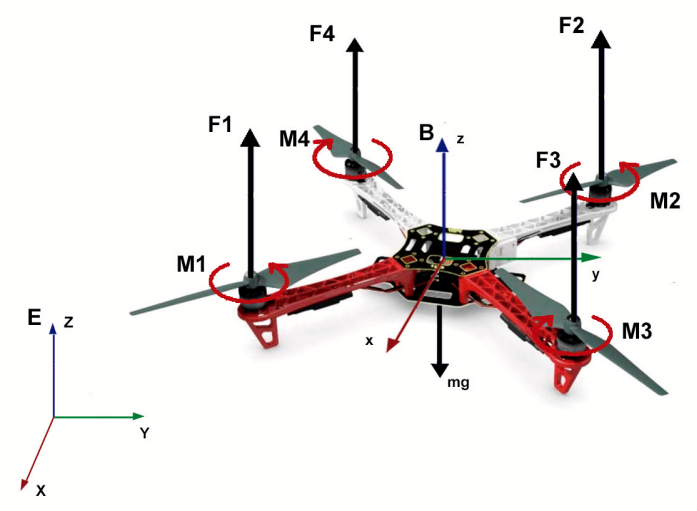

Fig. 3. Quad-rotor frame of reference.

1. Six states define the position of the system in the three dimensional space: Cartesian coordinate $(x, y, z)$ points to the center of gravity of the quadcopter and their time derivative $(u, v, w)$ defines the speed of the center of gravity relative to the earth.

2. Six states define the attitude of the system: Euler angles $(\phi, \theta, \psi)$ represent the roll, pitch, and yaw respectively and their time derivatives $(p, q, r)$ describe the rotation of the quad-rotor.

According to Fig. $3, F_{1}, F_{2}, F_{3}$, and $F_{4}$ are the forces applied to the rigid body by the motors. The effect of these forces produces roll angle $(\phi)$, pitch angle $(\theta)$, and yaw angle $(\psi)$. Four control inputs can be defined according to these four forces acting on the quad-rotors rigid body:

$$
\begin{aligned}
& U_{1}=F_{1}+F_{2}+F_{3}+F_{4}, \\
& U_{2}=\left(F_{2}-F_{4}\right) l, \\
& U_{3}=\left(F_{3}-F_{1}\right) l, \\
& U_{4}=M_{1}+M_{2}-M_{3}-M_{4} .
\end{aligned}
$$

$F_{i}$ and $M_{i}$ are the thrust and torque generated by rotor $i(i \in\{1,2,3,4\})$ and $l$ represents the length of the arm from the rotor to the center of mass. In [16] 
and [17], the Eq. (1) can be expressed in terms of equations, governing dynamics of the quadrotor as:

$$
\begin{aligned}
\ddot{x} & =\frac{U_{1}}{m}(\cos \phi \sin \theta \cos \psi+\sin \phi \sin \psi), \\
\ddot{y} & =\frac{U_{1}}{m}(\cos \phi \sin \theta \sin \psi-\sin \phi \cos \psi), \\
\ddot{z} & =\frac{U_{1}}{m} \cos \phi \cos \theta-g, \\
\ddot{\phi} & =\frac{U_{2}}{I_{x x}}+\dot{\theta} \dot{\psi}\left(\frac{I_{y y}-I_{z z}}{I_{x x}}\right)-\frac{J_{R}}{I_{x x}} \dot{\theta} \Omega_{R}, \\
\ddot{\theta} & =\frac{U_{3}}{I_{y y}}+\dot{\phi} \dot{\psi}\left(\frac{I_{z z}-I_{x x}}{I_{y y}}\right)-\frac{J_{R}}{I_{y y}} \dot{\phi} \Omega_{R}, \\
\ddot{\psi} & =\frac{U_{4}}{I_{z} z}+\dot{\phi} \dot{\theta}\left(\frac{I_{x x}-I_{y y}}{I_{z z}}\right)
\end{aligned}
$$

The position of the center of mass in the inertial coordinate system is $(x, y, z)$; $\phi, \theta$, and $\psi$ are the quad-rotor's attitude; $m, I_{x x}, I_{y y}$, and $I_{z z}$ are the mass and the moments of inertia respectively; $J_{R}$ and $\Omega_{R}$ are the moments of inertia and angular velocity of the propeller blades; and $g$ is the gravity.

\section{Quad-rotor Identification Model from Experimental Data}

The quad-rotor system can be treated as MIMO (Multiple Inputs and Multiple Outputs) system. See Fig. 4. The variables of interest are

1. 4 inputs (4 PWM signals),

2. 3 outputs (Euler angles).

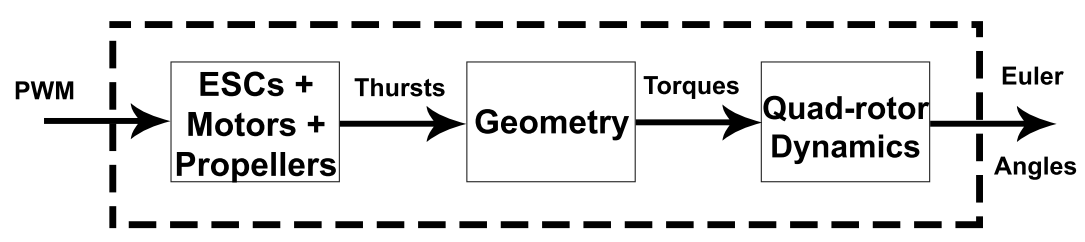

Fig. 4. Quad-rotor block diagram. 




Fig. 5. Flow diagram of the system identification.

\subsection{System Identification Algorithm}

The Fig. 5 shows the flow diagram of the identification algorithm. The first step to obtain the model is to prepare the data for system identification. Once the data is obtained from experiments, it has to be imported into Matlab environment. The data has to be represented in time and frequency domains using the iddata function. This function creates an iddata object containing a time-domain output signal $y$ and an input signal $u$. Also, the sample time of the experimental data has to be specified. Then a data selection is done to create independent data sets for estimation and validation.

The second step is to determine which model will be used to represent the linear system. The transfer function model is a numeric model that represents a linear system with fixed numerical coefficients. Those fixed coefficients are the numerator and denominator of the transfer function. For the Matlab System Identification Toolbox, the transfer function model describes the relationship between the inputs and outputs of a dynamic system using a ratio of polynomials.

The transfer function model, in continous time, has the following form:

$$
Y(s)=\frac{\operatorname{num}(s)}{\operatorname{den}(s)} U(s)
$$

where $Y(s)$ and $U(s)$ represent the Laplace transforms of the output and input, respectively. The equation (3) represents a single-input single-output (SISO) contionus transfer function. A multi-input multi-output (MIMO) transfer function has $n$ SISO transfer function depending on the input-output system. It has the following form:

$$
\begin{aligned}
G(s) & =\frac{\operatorname{num}(s)}{\operatorname{den}(s)}, \\
Y_{1}(s) & =G_{11} U_{1}(s)+G_{12} U_{2}(s)+\ldots+G_{1 n} U_{n}(s),
\end{aligned}
$$

where $G_{i j}(s)$ is the SISO transfer function between the $i^{\text {th }}$ output and $j^{\text {th }}$ input. The validation model is the last step to verify the accuracy of the model obtained. The validation data is used to check how the model system behaves and after that, do some refinements if the model requires it. 


\subsection{Obtaining the Models from System Identification}

Matlab has a System Identification Toolbox which is used to identify a linear model of the quad-rotor system. Experimental data was obtained and tested to get transfer functions from the roll and pitch axis. The best transfer functions that fit the data are the following:

From input $u_{1}$ to output $y_{1}$ :

$$
\begin{gathered}
-2.102 \times 10^{-10} \\
s^{11}+0.4659 s^{10}+0.8069 s^{9}+0.2817 s^{8}+0.1927 s^{7} \\
+0.05124 s^{6}+0.01715 s^{5}+0.003271 s^{4}+0.0005535 s^{3} \\
+7 \times 10^{-5} s^{2}+4.797 \times 10^{-6} s+3.605 \times 10^{-7}
\end{gathered}
$$

From input $u_{2}$ to output $y_{1}$ :

$$
\begin{gathered}
-9.898 \times 10^{-10} \\
\hline s^{11}+0.5932 s^{10}+1.051 s^{9}+0.4688 s^{8}+0.2964 s^{7} \\
+0.09754 s^{6}+0.03072 s^{5}+0.00695 s^{4}+0.001157 s^{3} \\
+0.0001635 s^{2}+1.199 \times 10^{-5} s+8.575 \times 10^{-7}
\end{gathered}
$$

From input $u_{3}$ to output $y_{2}$ :

$$
\begin{gathered}
\frac{-2.521 \times 10^{-8}}{s^{10}+0.3857 s^{9}+1.114 s^{8}+0.3282 s^{7}+0.4312 s^{6}+0.09092 s^{5}} \\
+0.069 s^{4}+0.009134 s^{3}+0.004089 s^{2}+0.0002373 s+4.474 \times^{-5}
\end{gathered}
$$

From input $u_{4}$ to output $y_{2}$ :

$$
\begin{aligned}
& \frac{-2.531 \times 10^{-8}}{s^{10}+0.3851 s^{9}+1.113 s^{8}+0.3276 s^{7}+0.4306 s^{6}+0.09073 s^{5}} \\
& \quad+0.06887 s^{4}+0.009111 s^{3}+0.004079 s^{2}+0.0002366 s+4.476 \times 10^{-5}
\end{aligned}
$$

From Matlab System Identification Toolbox, it is possible to obtain a pair of transfer functions that represent the dynamics of the roll and yaw axis. This model can be used on later stages to design a controller with better performance.

\section{Results}

The dataset obtained was divided in two sized data for both roll and pitch axis. First part was used to find the transfer functions of Eq. (5) and Eq.(6). The second part was used as validation data, in order to validate the model estimated.

\subsection{Model Validation}

The Fig. 6 shows the simulation of the roll and pitch axis from the estimated models respectively. The performance of the estimated models is quite good, although there are some parts in the figure where those models cannot represent the dynamics of the quad-rotor. This behavior is expected because the dynamics models are linear and the quad-rotor dynamics are normally represented by nonlinear models. 

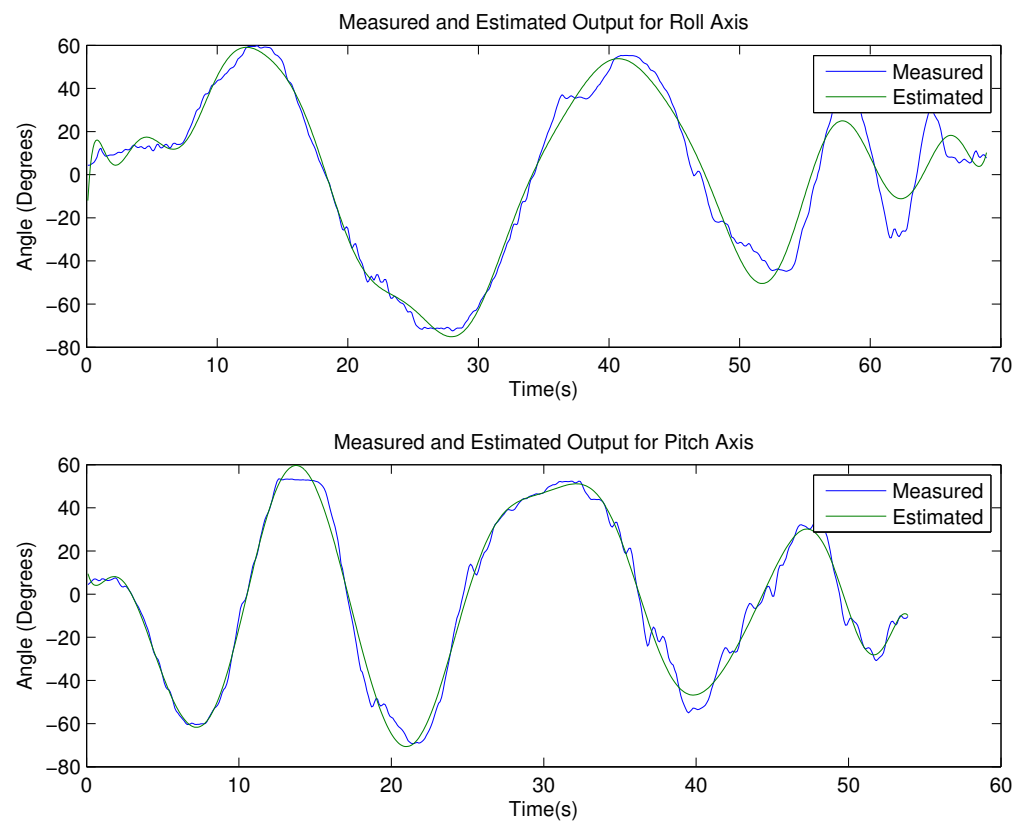

Fig. 6. Measured and estimated output for roll and pitch axis.

\section{Conclusion}

This paper presents a system identification method of a quad-rotor in X configuration based on a linear model. The estimated models represent the quad-rotor dynamics quite well, despite some irregularities in the estimated outputs system. It can be seen that the rapid changes in the quad-rotor dynamics, could be difficult to estimate for the linear models proposed in this paper.

The Matlab System Identification Toolbox is demonstrated to be a good tool for a quick modeling of a quad-rotor system. The transfer functions for the roll and pitch axis shows that a linear model can estimate the quad-rotor dynamics, even though the system have strong non-linearities.

Future research include the design of a controller based on the models proposed in this paper, the implementation of a neural network to obtain a nonlinear model which will improve the estimated output system, and the design of a stabilization control for the quad-rotor.

\section{References}

1. Valavanis, K.P., Vachtsevanos, G.J.: Definitions and Terminology. Handb. Unmanned Aer. Veh., pp. 1-3022 (2015) 
2. Hughes, V., Macnichol, E.F., Williams, F.C.: Technology and autonomous mechanisms in the Mediterranena: from ancient Greece to Byzantium. MCS.2014.2359589, pp. 110-119 (2014)

3. Valavanis, K.P., Vachtsevanos, G.J.: Aviaton history and unmanned flight. Handb. Unmanned Aer. Veh., pp. 1-3022 (2015)

4. Floreano, D., Wood, R.J.: Science, technology and the future of small autonomous drones. Nature 521, pp. 460-466, doi: 10.1038/nature14542 (2015)

5. Yang, J., Cai, Z., Lin, Q.: System identification of quadrotor UAV based on genetic algorithm. In: Proc. 2014 IEEE Chinese Guided Navigation Control Conf., pp. 2336-2340, doi: 10.1109/CGNCC.2014.7007533 (2014)

6. Chang, W.D.: Nonlinear system identification and control using a real-coded genetic algorithm. Appl Math Model 31, pp. 541-550, doi: 10.1016/j.apm.2005.11.024 (2007)

7. Wang, G., Xia, H., Yuan, X.: Modeling the yaw dynamics of an unmanned helicopter through modes partition method. Sci China Technol Sci 55, pp. 182-192, doi: 10.1007/s11431-011-4576-9 (2012)

8. Jin, H., Li, X., Zhang, X., Li, Y.: Study on the Modeling Methods of the Quadrotor Unmanned Helicopter. pp. 4855-4859 (2015)

9. Xiii, T., May, F.: Genetic fuzzy identification method for quadrotor UAVs. DEVELOPMENT OF MICROCONTROLLER-BASED SINGLE PHASE, Int J Eng pp. 237-240 (2015)

10. Sarioglu, A., Kural, A.: Modeling and ARX identification of a quadrotor MiniUAV. In: 2015 9th International Conference on Electrical and Electronics Engineering (ELECO), pp. 1196-1200, IEEE (2015)

11. Salameh, I.M., Ammar, E.M., Tutunji, T.A.: Identification of quadcopter hovering using experimental data. In: 2015 IEEE Jordan Conference on Applied Electrical Engineering and Computing Technologies (AEECT), pp. 1-6, IEEE (2015)

12. Wu, H., Sun, D., Zhou, Z.: Model Identification of a Micro Air Vehicle in Loitering Flight Based on Attitude Performance Evaluation. IEEE Trans., Robot 20, pp. 702-712 (2004)

13. Wu, J., Peng, H., Chen, Q.: RBF-ARX model-based modeling and control of quadrotor. In: 2010 IEEE International Conference on Control Applications, pp. 1731-1736, IEEE (2010)

14. Dongli, Y., Jianguo, Y., Xinmin, W., Qingbiao, X.: A Study of Information Fusion for UAV Based on RBF Neural Network. In: 2007 IEEE International Conference on Control and Automation, pp. 2839-2842, IEEE (2007)

15. Schreurs, R.J.A., Weiland, S., Tao, H.: Open loop system identification for a quadrotor helicopter system. In: IEEE Int Conf. Control Autom. ICCA, pp. 17021707, doi: 10.1109/ICCA.2013.6565145 (2013)

16. Bouabdallah, S., Murrieri, P., Siegwart, R.: Design and control of an indoor micro quadrotor. In: IEEE Int Conf Robot Autom 2004 Proceedings ICRA '04 2004 5, pp. 4393-4398, doi: 10.1109/ROBOT.2004.1302409 (2004)

17. Dydek, Z.T., Annaswamy, A.M., Lavretsky, E.: Adaptive control of quadrotor UAVs: A design trade study with flight evaluations. IEEE Trans Control Syst Technol 21, pp. 1400-1406, doi: 10.1109/TCST.2012.2200104 (2013)

18. Dong, W., Gu, G.Y., Zhu, X., Ding, H.: A high-performance flight control approach for quadrotors using a modified active disturbance rejection technique. Rob Auton Syst 83, pp. 177-187, doi: 10.1016/j.robot.2016.05.005 (2016) 